\title{
NOAA-AVHRR Orbital Drift Correction From Solar Zenithal Angle Data
}

\author{
José A. Sobrino, Yves Julien, Mariam Atitar, and Françoise Nerry
}

\begin{abstract}
This paper presents a new method for NOAA's (National Ocean and Atmospheric Administration) orbital drift correction. This method is pixel-based, and in opposition with most methods previously developed, does not need explicit knowledge of land cover. This method is applied to AVHRR (Advanced Very High Resolution Radiometer) channel information, and relies only on the additional knowledge of solar zenithal angle (SZA) and acquisition date information. In a first step, anomalies in SZA and channel time series are retrieved, and screened out for anomalous values. Then, the part of the parameter anomaly which is explained by SZA anomaly is removed from the data, to estimate new parameter anomalies, and this iteratively until the influence of SZA anomalies is totally removed from the parameter data. This correction has been applied to bimonthly AVHRR data provided by the GIMMS group (Global Inventory Modeling and Mapping Studies), covering Africa from November 2000 to December 2006. NDVI and LST (Land Surface Temperature) have been estimated from raw and corrected data, and averaged over homogeneous vegetation classes. Differences between raw and corrected averaged parameters show an improvement in the quality of the data. In order to validate this method, a whole week (10 to 17 July 2004) of METEOSAT SEVIRI (Spinning Enhanced Visible and InfraRed Imager) data have been used, from which LST have been estimated using a similar method to the one used to retrieve LST from AVHRR data. The comparison between both platforms at the same time of acquisition shows good concordance.
\end{abstract}

Index Terms-Land surface temperature (LST), national ocean and atmospheric administration (NOAA) advanced very high resolution radiometer (AVHRR), normalized vegetation difference index (NDVI), orbital drift, solar zenithal angle (SZA).

\section{INTRODUCTION}

A VHRR (Advanced Very High Resolution Radiometer) sensor, embarked on NOAA (National Oceanic and Atmospheric Administration) platforms since the early 1980s, has provided the scientific community with highly valuable data about our planet. Although NOAA satellite series had been designed for ocean and atmosphere survey, one of its

Manuscript received November 14, 2007; revised February 21, 2008. Current version published November 26, 2008. This work was supported in part by the European Union (EAGLE, Project SST3-CT-2003-502057) and the TERMASAT project (Ministerio de Educación y Ciencia, project ESP200507724-C05-04).

J. A. Sobrino, Y. Julien, and M. Atitar are with the Global Change Unit, Department of Earth Physics and Thermodynamics, University of Valencia,46100 Burjassot, Spain (e-mail: sobrino@uv.es; yves.julien@uv.es; mariam.atitar@uv.es)

F. Nerry is with the École Nationale Supérieure de Physique de Strasbourg (ENSPS), Laboratoire des Sciences de l'Image, de l'Informatique et de la Télédétection (LSIIT)/TRIO, 67412 Illkirch, France (e-mail: nerry @ 1siit. u-strasbg.fr).

Color versions of one or more of the figures in this paper are available online at http://ieeexplore.iee.org.

Digital Object Identifier 10.1109/TGRS.2008.2000798 unforeseen applications has been earth observation. This is due to the choice of the bands of the AVHRR instrument: its visible (red) and near-infrared bands correspond, respectively to vegetation absorption and reflection [1]. From these properties, a normalized vegetation difference index (NDVI) has been designed [2], [3], in order to characterize the photosynthetic activity of the observed area, a proxy to vegetation health. In addition to these two bands, the AVHRR sensor has one band in middle infrared, usually dedicated to cloud detection (and generally not available in public data sets), and two bands in thermal infrared, from which land surface temperature (LST) can be estimated by split-window methods [4]. The combined use of NDVI and LST data can help understand better the nature of the changes undergone by the observed vegetation [5].

However, few studies have used both data (NDVI and LST) for vegetation studies [5], due to a major flaw in the data retrieval. NOAA platforms do not include any system to stabilize their orbit, which means that although the satellites have been placed successfully on their nominal orbit (heliosynchronous, 13:30 or 14:00 UMT depending on the afternoon satellites), they derived slowly from those orbits [6]. This derive, also called orbital drift, implicates that instead of taking images of the earth at the same hour each day over a given area, the images have been taken progressively later in the afternoon. Since the satellite had been placed on an orbit corresponding to maximum thermal emission, this means that the thermal emission was smaller, and thus introduces an artificial cooling of the images over time [6]. This orbital drift effect prevents from the use of time series of AVHRR retrieved LST for earth observation studies. This orbital drift effect can also be evidenced in NDVI time series, depending on vegetal cover.

Several methods have been developed to correct this orbital drift, generally based on solar zenithal angle (SZA). Reference [7] developed a method for orbital drift correction in radiometric temperature data, which consists in a previous classification of the study area, over which LST and SZA time series are averaged, and anomalies in LST are then adjusted to SZA anomalies to eliminate from LST time series the part that can be explained by SZA anomalies. Reference [8] developed a model based on land occupation to estimate a daily temperature cycle for each pixel, from which they correct the retrieved LST from the hour difference due to the orbital drift. This model has been developed over latitude bands of $5^{\circ}$ for temperate areas, leading to artificial discontinuities at global scale. Reference [9] developed another model taking into account the illumination geometry over the observed scene to determine the proportion of shadows integrated by the sensor, which varies with the image acquisition time. Reference [10] used the 
Empirical Mode Decomposition (EMD) technique to remove SZA influences on NDVI, by identifying the correlated modes of NDVI and SZA. Reference [11] corrected red and near infrared AVHRR channels estimating directional reflectances from POLDER data (POLarisation and Directionality of the Earth's Reflectance).

All the methods described above (with the exception of the EMD technique) imply explicit knowledge of the observed pixel to carry out the correction. These techniques cannot be applied when the study aims precisely at pixel characterization (for instance when changes are investigated through time series analysis). Thus, a correction of the time series without a priori knowledge of pixel characteristics is needed. This study presents a new method to correct AVHRR channel information from the orbital drift, in order to make possible time series analyzes from AVHRR LST. However, building such time series is beyond the scope of this paper.

\section{DATA}

This study uses GIMMS (Global Inventory Modeling and Mapping Studies) data [12] provided by M. E. Brown. These data cover Africa for the period ranging from November 2000 to December 2006, corresponding to NOAA-16 (November 2000 to December 2004) and NOAA-17 (January 2005 to December 2006) activity periods. These data are quasi 15 -day composites, and have a spatial resolution of $8 \mathrm{~km} .8 \mathrm{~km}$ spatial resolution was obtained by forward mapping of the satellite data in NOAA level 1B format [13] to the output bin closest to the center location of each $8 \mathrm{~km}$ grid cell, and calibration values were applied to each channel [14]. At this resolution, land cover is mainly heterogeneous, however, assuming a good geo-referencing of the data by the GIMMS group, the observed pixels correspond to the same area. Therefore, pixels may include heterogeneous land covers, though their proportion in the pixel is stable throughout the considered time series. The data were then composited following the NDVI MVC (Maximum Value Compositing) technique [15], which means that daily NDVI images were calculated and aggregated in quasi 15 day composites by selecting the day for which maximum NDVI value is reached during each compositing period for each pixel. This reference day for the given pixel is also chosen for compositing the rest of the available channels. The composite character of the data does not have any influence on the correction, since acquisition date is provided for each pixel. Moreover, sampling land cover annual cycle at 15 day resolution assumes land cover to be stationary over 15 days, which may not be the case throughout the year. For example, periods of rapid change such as vegetation onset and leaf fall may occur at a quicker rate. However, these events have an interannual variability which can be of the order of one month, which makes a smaller compositing period unnecessary.

The data used in this work are the following:

- Channels 1 and 2, corresponding to red and infrared reflectances, respectively centered at 0.6 and $0.8 \mu \mathrm{m}$.

- Channels 4 and 5, corresponding to radiometric temperatures, respectively centered at 11 and $12 \mu \mathrm{m}$.
- SZA and SCA data, corresponding, respectively to solar zenithal and viewing angles, in radians.

- DAY data, which informs of the day of acquisition of the data, expressed in Julian days.

These GIMMS images are not corrected for atmospheric effects; however, this point is not critical, since the aim of this study consists in determining the feasibility of correcting NOAA-AVHRR orbital drift. Thus, no atmospheric correction has been applied to the data. Of course, in order to obtain adequate biophysical parameters from the images, such a correction is unavoidable.

The times series length, from November 2000 to December 2006 , covers the whole activity period of NOAA-16, as well as part of NOAA-17 activity period (148 dates). Therefore, the data cover the whole orbital drift of NOAA-16 as well as its replacement with NOAA-17. The effects of these events are an artificial decrease of retrieved LST throughout the active period of NOAA-16 (until December 2004), followed by a sudden increase in LST due to NOAA-16 replacement by NOAA-17, which then started to drift. Therefore, the data extent covers all the changes occurring because of the orbital drift, which means that these data are suitable for carrying out an orbital drift correction.

\section{Methodology}

The methodology applied in this work relies on the assumption that most of the errors due to NOAA orbital drifts are related to solar zenithal angle information [7]. This relation is supposed to be satellite dependent, since all NOAA platforms were not placed on the same nominal orbit, and pixel dependent, since surface land cover influences the magnitude of the error in each parameter [6].

\section{A. Anomalies}

First, solar zenithal angle time series (SZA) are estimated for fixed overpass time at 13:30 for each pixel [16], using day of acquisition information (DAY data). This time has been chosen since it corresponds to some of NOAA satellite nominal orbits, and it allows easy validation by comparison with geostationary data. For easier comprehension, these SZA time series are referred to as nominal SZA in the rest of the manuscript. Then, SZA anomalies are calculated as the difference between retrieved (SZA data) and nominal SZA time series.

As for channel data pixel anomalies, the procedure for anomaly calculation is the following: first, an average year of data is calculated for each pixel, by averaging successively for each compositing period over the 6 years of data. Thus, 24 average images corresponding to 1-15 January, 16-31 January, 1-15 February, etc. are obtained, creating the average year mentioned above. Then, those composite average values are subtracted from the whole time series (148 images), each composite average being subtracted from the corresponding composite pixel values, creating channel data anomalies for each pixel.

Finally, since residual clouds or errors in SZA are present in the data, outliers in anomalies have to be screened out. To this 
end, a histogram of the whole time series is calculated, setting bin width to one standard deviation around the mean value of the time series. The values located in the extreme bins of the histogram are labeled as outliers if any bin closest to the mean value is empty. This aims at identifying the outliers, assuming a Gaussian distribution of the anomalies. This way, errors in solar zenithal angle determination, as well as cloud contaminated values in channel data, are automatically rejected from the anomaly time series, and do not compute in the correction of the orbital drift for every channels.

\section{B. Fitting Procedure}

In order to remove SZA anomaly influence on parameter $\left(\mathrm{Ch}^{i}, i=1,2,4\right.$, or 5$)$ anomaly $\left(\mathrm{Chi}_{\text {an }}\right)$, an iterative procedure has been developed. This procedure consists first in a linear regression between parameter anomaly and SZA anomaly $\left(\mathrm{SZA}_{\mathrm{an}}\right)$

$$
\mathrm{Chi}_{\mathrm{an}}=a+b \cdot \mathrm{SZA}_{\mathrm{an}} .
$$

If this linear regression is statistically significant at $95 \%$ confidence level, then a new parameter time series $\left(\mathrm{Chi}^{1}\right)$ is calculated from the uncorrected one $\left(\mathrm{Chi}^{0}\right)$

$$
\mathrm{Chi}^{1}=\mathrm{Chi}^{0}-\left(a+b \cdot \mathrm{SZA}_{\mathrm{an}}\right) .
$$

Then, new parameter anomalies are calculated from $\mathrm{Chi}^{1}$, and a new linear regression is carried out from the same SZA anomalies. This iterative procedure finishes when the difference in the whole time series standard deviation between two iterations gets under a given threshold, fixed at 0.0001 for channels 1 and 2 reflectances, and $0.01 \mathrm{~K}$ for channels 4 and 5 radiometric temperatures.

\section{Limitations}

This method for NOAA orbital drift correction does not take into account the influence of other factors than SZA effect in image acquisition, meaning that the atmospheric absorption is still present in the data. The approach presented here assumes that atmospheric absorption and SZA effect are decorrelated, which is only partially true. This means that for an adequate data set correction, this approach should be applied to atmospherically corrected time series. However, building a time series of exploitable LST time series is beyond the scope of this paper.

Moreover, subtracting the average year of data to estimate anomalies may not correspond to parameter values without orbital drift. This drawback could be eliminated using ground measurements at a given date for various sites. However, the time series of corrected parameter on one hand and of parameter without orbital drift on the other hand differ only by a constant. Therefore, corrected parameter time series are temporally consistent and can be used for change analysis.

Another limitation of this method is the availability of various years of data from the same satellite platform, since the anomalies have to be estimated from a sufficiently long period of time to have statistical meaning.

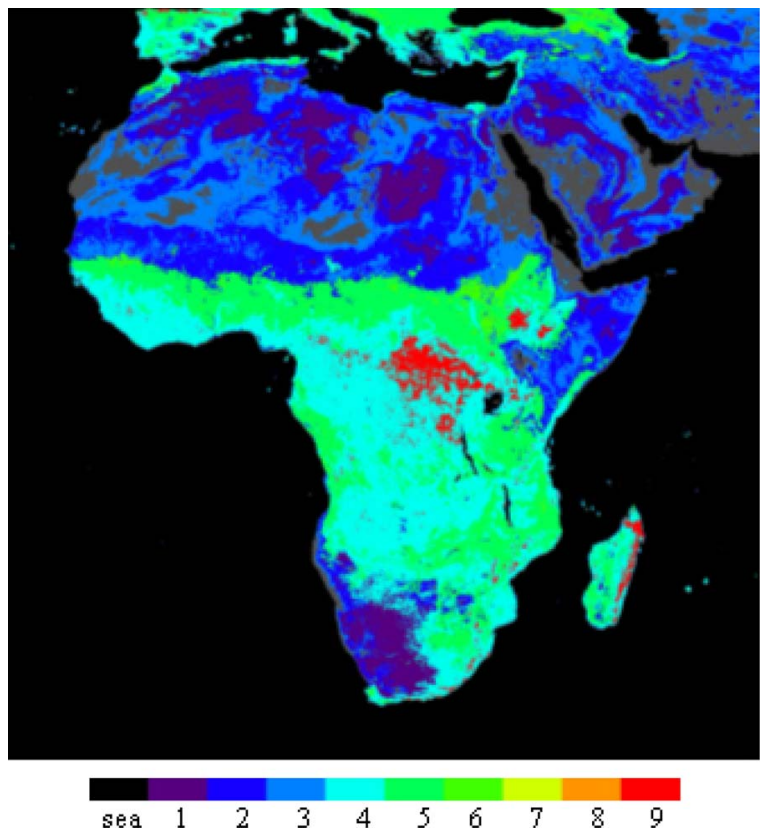

Fig. 1. Classification of the African continent vegetation. Dark grey areas correspond to areas with insufficient NDVI data.

\section{RESUlTS}

NDVI and land surface temperature (LST), which are essential for vegetation monitoring, have been estimated from channel information. NDVI is calculated as the normalized difference between channel 2 and channel 1 information [2]. LST estimation algorithm is based on a split-window method, and is carried out through previous estimations of emissivity and total atmospheric vapor content [4].

An NDVI based classification has been calculated from corrected NDVI time series, following the methodology presented in [17], [18]. This classification is based on the calculation of an average NDVI year, from which average NDVI values (MN) and variation coefficients (VC) are calculated. This classification is presented in Fig. 1, and includes 10 classes, defined as follow: $0-$ Sea; $1-$ (MN $<0.2$ and $\mathrm{VC}<0.331 ; 2-\mathrm{MN}$ $<0.2$ and $0.33 \leq \mathrm{VC}<0.66 ; 3-\mathrm{MN}<0.2$ and $0.66<$ $\mathrm{VC} ; 4-0.2 \leq \mathrm{MN}<0.5$ and $\mathrm{VC}<0.33 ; 5-0.2 \leq \mathrm{MN}<0.5$ and $0.33 \leq \mathrm{VC}<0.66 ; 6-0.2 \leq \mathrm{MN}<0.5$ and $0.66<\mathrm{VC}$; $7-0.5 \leq \mathrm{MN}$ and $0.66<\mathrm{VC} ; 8-0.5 \leq \mathrm{MN}$ and $0.33 \leq \mathrm{VC}<$ 0.66 ; and $9-0.5 \leq \mathrm{MN}$ and $\mathrm{VC}<0.33$. The aim of the use of this classification is to average the corrected and uncorrected parameters over homogeneous classes, in order to observe the effects of the correction over extended areas.

This classification divides the arid areas in 3 classes (1, 2, and 3), and the vegetated areas in the remaining 6 classes. Classes 7 and 8 are absent of the image, due to the very small amount of pixels attributed to these classes (respectively 0 and 76 pixels). Differences between corrected and uncorrected NDVI and LST have been averaged over classes containing a significant number of pixels (greater than 100 pixels)-all classes except classes 7 and 8 . These differences are presented in Fig. 2.

From a general point of view, the correction is more important at the end of the satellite life-period than at its beginning for 
SOBRINO et al: NOAA-AVHRR ORBITAL DRIFT CORRECTION FROM SOLAR ZENITHAL ANGLE DATA

4017
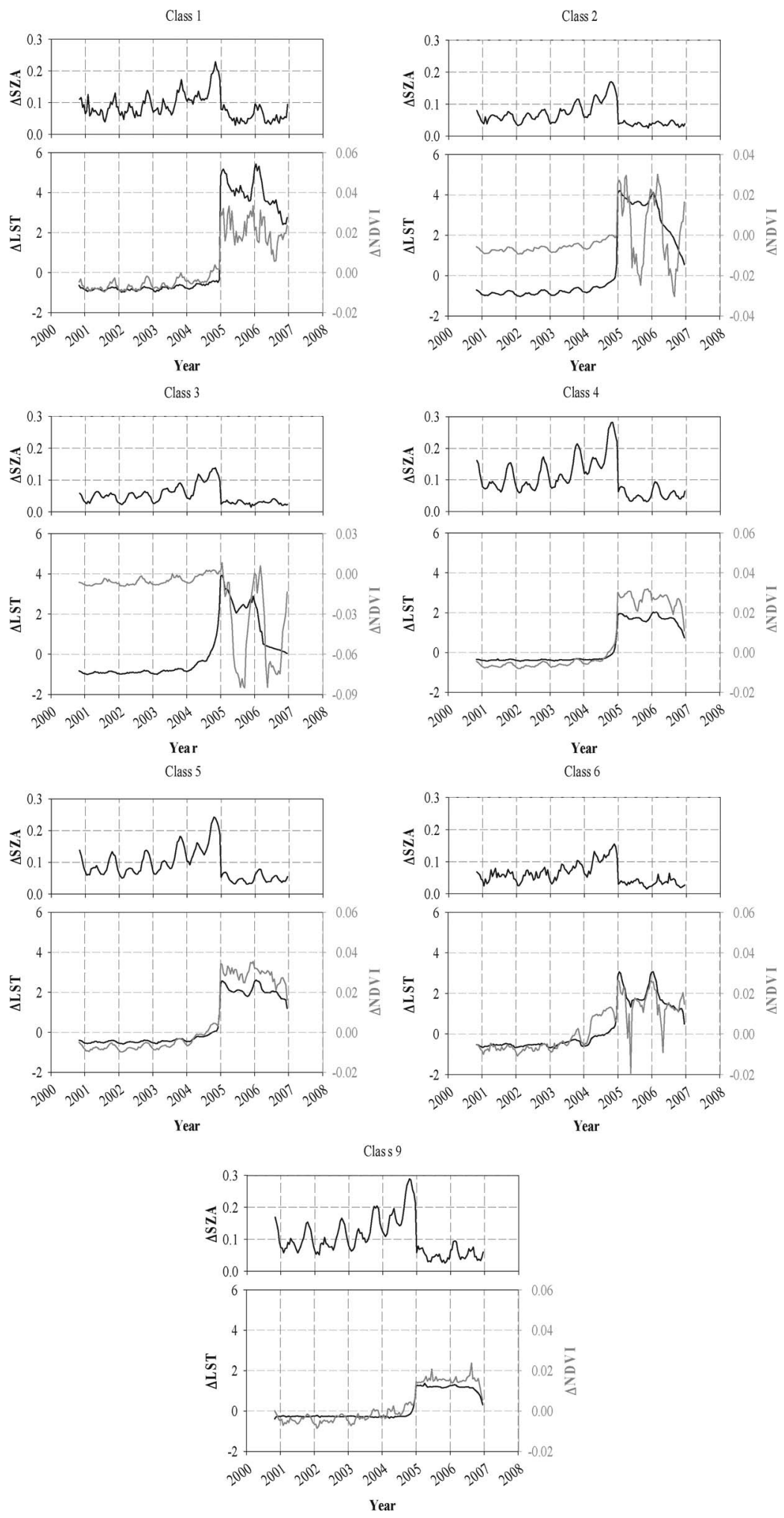

Fig. 2. SZA anomalies ( $\Delta$ SZA - upper graph) for all classes, and differences between corrected and uncorrected LST ( $\Delta$ LST-lower graph, left axis, grey) and NDVI ( $\triangle$ NDVI-lower graph, right axis, black) time series for all significant classes.

all classes, corresponding to decreases in both LST and NDVI uncorrected values, except in the case of NDVI for classes 2 , 4, and 6. As one can easily observe in Fig. 2, correction amplitudes vary with class number, meaning that amplitudes are specific to vegetation types. Corrections are different for all classes. Correction also depends on the satellite platform, the correction being generally more important for NOAA-17 than for NOAA-16. This difference for mean LST and NDVI values between both platforms is due to the lack of intercalibration between both platforms (M. E. Brown, personal communication).

Authorized licensed use limited to: IEEE Xplore. Downloaded on January 16, 2009 at 04:04 from IEEE Xplore. Restrictions apply. 
This also explains why the mean corrected values are positive for one of the satellite, and negative for the other one, since this lack of intercalibration affects the anomaly calculation.

NDVI corrections are bigger for vegetated areas (classes 5, 6 and 9), corroborating previous analyzes that orbital drift affects little desert areas as regards NDVI values [19]. Regarding LST correction, its amplitude is higher for arid areas (classes 1,2, and 3), due to the high amplitude of the LST daily cycle over those areas.

Seasonal and annual components can be identified the time series of difference between corrected and uncorrected NDVI and LST. This is due to the presence of seasonal and annual components in SZA anomalies, due to the hour difference between AVHRR data acquisition and 13:30 sun hour. Simple difference between time series of SZA estimated at 13:30 and 15:00 using [16] show this seasonal pattern (not shown). Therefore, these components are part of the orbital drift error, and adequately corrected by the procedure presented above.

\section{VALIDATION AND DISCUSSION}

In order to validate the results presented above, the authors decided to use MSG (Meteosat Second Generation) SEVIRI (Spinning Enhanced Visible and Infrared Imager) data retrieved during the SPARC (SPectra bARrax Campaign) campaign of 2004 (10 to 17 July). The main objective of the Spectra Barrax Campaigns was to collect coincident in situ data over the Barrax site, about $20 \mathrm{~km}$ west of Albacete, Spain, with CHRIS/PROBA multiangular and multispectral in order to address the following critical issues in the Earth-Explorer SPECTRA Phase A study: validation of BRDF forward models, validation of SPECTRA geophysical parameter retrieval algorithms, development and testing of atmospheric correction algorithms for multiview images, and validation of multiple view image coregistration algorithms. Additional data from ROSIS, HYMAP, and AHS airborne sensors, flying simultaneously with CHRIS overpasses, provided detailed images for validation of CHRIS data, particularly in the spectral domain. Moreover, multiangular airborne HYMAP and AHS data were acquired, with high spectral and spatial resolution, and then both spectral and angular domains can be exploited with the combined data set. Detailed soil/vegetation and atmospheric measurements complete the SPARC data set, and data from other satellites (MERIS, SEVIRI, SPOT, Landsat) were collected as well, to address scaling issues.

The collected MSG data consist of a whole week of SEVIRI images, with all bands at 15 minute intervals. From these data, land surface temperatures have been estimated for images acquired at 13:30 (in concordance with AVHRR corrected images), using a split-window algorithm similar to the one applied above [20].

Using DAY information from GIMMS data, two SEVIRI composites were created corresponding to GIMMS composites (July15a and July15b). Then, SEVIRI LSTs were resampled to fit GIMMS image projection (Albers). Finally, the corresponding GIMMS and SEVIRI LSTs were compared over the common days of the respective data sets. Since NOAA-AVHRR sensor thermal bands have a saturation value between 320 and
TABLE I

DIFFERENCES BETWEEN SEVIRI AND GIMMS LST AVERAGED OVER Homogeneous Vegetation Classes (SEe Fig. 1)

\begin{tabular}{c|cc|cc}
\hline \hline class & \multicolumn{2}{|c|}{ Number of pixels } & \multicolumn{2}{|c}{$\Delta$ LST } \\
& $\mathbf{A}$ & $\mathbf{B}$ & $\mathbf{A}$ & $\mathbf{B}$ \\
\hline $\mathbf{1}$ & 8612 & 608 & -3.51 & -3.89 \\
$\mathbf{2}$ & 12909 & 1877 & -2.79 & -6.50 \\
$\mathbf{3}$ & 4667 & 2118 & -4.29 & -8.03 \\
$\mathbf{4}$ & 24267 & 14489 & -1.57 & -1.70 \\
$\mathbf{5}$ & 19056 & 9214 & -1.20 & -2.42 \\
$\mathbf{6}$ & 951 & 584 & -2.19 & -4.25 \\
$\mathbf{7}$ & $/$ & $/$ & $/$ & $/$ \\
$\mathbf{8}$ & 5 & 13 & 2.45 & 0.78 \\
$\mathbf{9}$ & 813 & 1629 & -0.83 & 0.56 \\
A and B columns refer to composite images 15a and 15b corresponding \\
respectively to 1-15 and 16-31 July 2004. \\
ALST is the difference between LST retrieved from SEVIRI sensor and \\
corrected LST retrieved from GIMMS data.
\end{tabular}

$330 \mathrm{~K}$, pixels with LST value higher than $320 \mathrm{~K}$ were removed from the comparison, as well as pixels with LST value lower than $273 \mathrm{~K}$, in order to screen out residual clouds in the images.

The averaged difference between SEVIRI and GIMMS corrected LST over Africa is of $-2.32 \mathrm{~K}$. Since algorithm accuracies are, respectively of $1.7 \mathrm{~K}$ for NOAA-AVHRR and $2.4 \mathrm{~K}$ for MSG-SEVIRI at high view angle, this result is satisfying, being lower than their quadratic sum $(\delta T=2.9 \mathrm{~K})$.

The classification described previously has been used to average LST differences between SEVIRI and GIMMS over homogeneous vegetation classes. The results are presented Table I. Those results show that the vegetated classes (class number 4, 5, 8, and 9) exhibit generally lower LST differences. Since class 8 includes too few pixels to be statistically significant, those values will not be discussed. Classes 4, 5, and 9 correspond to stable vegetated areas, while the classes with higher averages correspond to arid and semiarid areas. This means that algorithms for LST estimation from SEVIRI and GIMMS data agree better on vegetated areas (with accuracies better than $\delta T$ ) than on arid and semiarid areas. This behavior could be due to angular effects. However, explaining the reasons for such discrepancies is beyond the scope of this paper.

One can also observe that the differences are lower for the first composite (A) than for the second (B), probably due to the fact that only two days of SEVIRI data were available to create this composite.

Since NOAA-16 had been launched on the 21st of September 2000 , this satellite had already derived significantly from its nominal orbit by mid-July 2004. Thus, reaching such a good agreement between MSG SEVIRI and NOAA-16 AVHRR data after orbital drift correction shows the validity of the correction method described.

\section{CONCLUSION}

The method presented above shows a good correction of NOAA's orbital drift, which is evidenced by a progressive increase of the correction amplitude trough each platforms lifetime. Additionally, this method performs an intercalibration within sensors, observable especially in areas with low vegetation cover. Due to the difficulty of acquiring ground data over extended areas to validate this correction, simultaneous 
observations by SEVIRI sensor have been compared with corrected data, and show a good agreement between observations.

This correction will be applied to similar data over the rest of the continents to study vegetation behavior, and will also be applied to the whole Pathfinder AVHRR Land data set, although information of acquisition day and SZA data are not freely available at the moment of redaction of this paper.

\section{ACKNOWLEDGMENT}

The authors wish to thank Molly E. Brown from the GIMMS group for providing the GIMMS data used in this work, as well as valuable information regarding these data.

\section{REFERENCES}

[1] D. M. Gates, Biophysical Ecology. New York: Springer-Verlag, 1980. $611 \mathrm{p}$.

[2] J. W. Rouse, R. H. Haas, J. A Schell, and D. W Deering, "Monitoring vegetation systems in the great plains with ERTS," in Proc. 3rd ERTS Symp., 1973, pp. 309-317. NASA SP-351 I.

[3] C. J Tucker, "Red and photographic infrared linear combinations for monitoring vegetation," Remote Sens. Environ., vol. 8, no. 2, pp. 127-150, May 1979.

[4] J. A. Sobrino and N. Raissouni, "Toward remote sensing methods for land cover dynamic monitoring: Application to Morocco," Int. J. Remote Sens., vol. 21, no. 2, pp. 353-366, Jan. 2000.

[5] R. Nemani and S. Running, "Land cover characterization using multitemporal red, near-IR, and thermal-IR data from NOAA/AVHRR," Ecol. Appl., vol. 7, no. 1, pp. 79-90, Feb. 1997.

[6] J. C. Price, "Timing of NOAA afternoon passes," Int. J. Remote Sens., vol. 12, no. 1, pp. 193-198, Jan. 1991.

[7] G. G. Gutman, "On the monitoring of land surface temperatures with the NOAA/AVHRR: Removing the effect of satellite orbit drift," Int. J. Remote Sens., vol. 20, no. 17, pp. 3407-3413, Nov. 1999.

[8] M. Jin and R. E. Treadon, "Correcting the orbit drift effect on AVHRR land surface skin temperature measurements," Int. J. Remote Sens., vol. 24, no. 22, pp. 4543-4558, Nov. 2003.

[9] A. C. T. Pinheiro, J. L. Privette, R. Mahoney, and C. J. Tucker, "Directional effects in a daily AVHRR land surface temperature dataset over Africa," IEEE Trans. Geosci. Remote Sens., vol. 42, no. 9, pp. 1941-1954, Sep. 2004.

[10] J. E. Pinzón, M. E. Brown, and C. J. Tucker, "EMD correction of orbital drift artifacts in satellite data stream," in The Hilbert-Huang Transform and Its Applications, N. E. Huang and S. S. P. Shen, Eds. Singapore: World Scientific, 2005.

[11] C. Bacour, F.-M. Bréon, and F. Maignan, "Normalization of the directional effects in NOAA-AVHRR reflectance measurements for an improved monitoring of vegetation cycles," Remote Sens. Environ., vol. 102, no. 3/4, pp. 402-413, Jun. 2006.

[12] C. J. Tucker, J. E. Pinzon, M. E. Brown, D. A. Slayback, E. W. Pak, R. Mahoney, E. F. Vermote, and N. El Saleous, "An extended AVHRR 8-km NDVI dataset compatible with MODIS and SPOT vegetation NDVI data," Int. J. Remote Sens., vol. 26, no. 20, pp. 4485-4498, Oct. 2005.
[13] K. B. Kidwell, Polar oorbiter data user's guide (TIROS-N, NOAA-6, NOAA-7, NOAA-8, NOAA-9, NOAA-10, NOAA-11, NOAA-12, NOAA-14). Washington D.C.: Nat. Ocean. Atmos. Admin., 1998.

[14] E. Vermote and Y. J. Kaufman, "Absolute calibration of AVHRR visible and near-infrared channels using ocean and cloud views," Int. J. Remote Sens., vol. 16, no. 13, pp. 2317-2340, 1995.

[15] B. N. Holben, "Characteristics of maximum-value composite images from temporal AVHRR data," Int. J. Remote Sens., vol. 7, no. 11, pp. 14171434, Nov. 1986.

[16] M. Iqbal, An Introduction to Solar Radiation, F. A. Kasten, Ed. Toronto, ON, Canada: Academic, 1983.

[17] L. Morales, G. Castellaro, J. A. Sobrino, and J. El Kharraz, "Land cover dynamic monitoring in the region of Coquimbo (Chile) by the analysis of multitemporal NOAA-AVHRR NDVI images," in Proc. ISPRS Conf. Istanbul, Turquia: Commission VI, Jul. 12-23, 2004.

[18] Y. Julien, J. A. Sobrino, and W. Verhoef, "Changes in land surface temperatures and NDVI values over Europe between 1982 and 1999," Remote Sens. Environ., vol. 103, no. 1, pp. 43-55, Jul. 2006.

[19] R. K. Kaufmann, L. Zhou, Y. Knyazikhin, N. V. Shabanov, R. B. Myneni, and C. J. Tucker, "Effect of orbital drift and sensor changes on the time series of AVHRR vegetation index data," IEEE Trans. Geosci. Remote Sens., vol. 38, no. 6, pp. 2584-2597, Nov. 2000.

[20] J. A. Sobrino and M. Romaguera, "Land surface temperature retrieval from MSG1-SEVIRI data," Remote Sens. Environ., vol. 92, no. 2, pp. 247-254, Aug. 2004

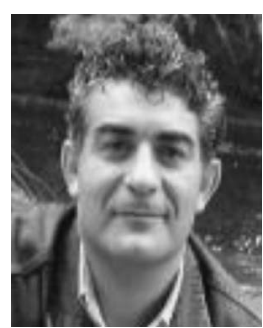

José A. Sobrino is Professor of physics and remote sensing and Head of the Global Change Unit, University of Valencia, Valencia, Spain. He is the author of more than 100 papers. He is the Coordinator of the European projects WATERMED and EAGLE. He was the Chairperson of First and Second International Symposium of the Recent Advances in Quantitative Remote Sensing. His research interest include atmospheric correction in visible and infrared domains, the retrieval of emissivity and surface temperature from satellite images, and the development of remote sensing methods for land cover dynamic monitoring.

Dr. Sobrino has been a member of Earth Science Advisory Commitee (ESAC) of European Space Agency (ESA), since November of 2003.

Yves Julien, photograph and biography not available at the time of publication.

Mariam Atitar, photograph and biography not available at the time of publication.

Françoise Nerry, photograph and biography not available at the time of publication. 